\title{
Disaccharide nucleosides as inhibitors of DNA repairing enzymes
}

\author{
M. Drenichev ${ }^{*}$, A. Komarova ${ }^{2,3}$, N. Dyrkheeva ${ }^{2}$, I. Kulikova ${ }^{1}$, V. Oslovsky ${ }^{1}$, \\ O. Zakharova ${ }^{2}$, A. Zakharenko², S. Mikhailov ${ }^{1}$, O. Lavrik ${ }^{2}$ \\ ${ }^{1}$ Engelhardt Institute of Molecular Biology, Russian Academy of Sciences, Moscow, Russia \\ ${ }^{2}$ Institute of Chemical Biology and Fundamental Medicine, SB RAS, Novosibirsk, Russia \\ ${ }^{3}$ Department of Natural Sciences, Novosibirsk State University, Novosibirsk, Russia \\ *e-mail:mdrenichev@mail.ru
}

Key words: disaccharide nucleosides, poly(ADP-ribose)polymerase 1, tyrosyl-DNA phosphodiesterase 1, DNA repair

Motivation and Aim: Numerous drugs have been developed, which have or resemble nucleosidic structure. Disaccharide nucleosides is a group of natural compounds forming poly(ADP-ribose) (PAR) and found in tRNA, antibiotics, and other physiologically active compounds [1]. Earlier we have developed the synthetic methodology for the synthesis of 2'-O- $\alpha$-D-ribofuranosyladenosine, a monomeric unit of PAR, which is an important biopolymer, participating in DNA repair [2]. Therefore, chemical synthesis of disaccharide analogues may be an advantageous area for the discovery of a novel compounds inhibiting DNA-repairing enzymes to increase the effectivity of anticancer therapy.

Methods and Algorithms: We have synthesized a series of disaccharide purine and pyrimidine nucleosides by the formation of an $O$-glycosidic bond between a nucleoside carrying one free hydroxyl group and an activated monosaccharide. All the compounds were characterized by NMR and UV spectroscopy and by LC-APCI and LC-HRMS. Inhibitory effect of disaccharide nucleosides was studied on two DNA-repairing enzymes - poly(ADP-ribose)polymerase 1 (PARP-1) and tyrosyl-DNA phosphodiesterase 1 (Tdp-1). PARP-1 inhibitory assay was performed using $\left[{ }^{3} \mathrm{H}\right]-\mathrm{NAD}^{+}$. Tdp-1 activity was measured using real-time fluorescence assay in the presence of fluorophorequencher containing oligonucleotides.

Results: In the series of disaccharide nucleosides pyrimidine derivatives were found to be effective Tdp-1 inhibitors, with $\mathrm{IC}_{50}$ being in low micro molar range, and weak PARP-1 inhibitors, with $\mathrm{IC}_{50}$ being $\sim 10^{-5} \mathrm{M}$. Disaccharide nucleosides did not demonstrate cytotoxic effects at concentration up to $1 \mathrm{mM}$ against human cell lines.

Conclusion: Thus, the obtained results have revealed disaccharide nucleosides as a promising class of compounds, inhibiting key DNA-repairing enzymes: PARP and Tdp-1. Acknowledgements: Supported by Russian Scientific Foundation (grant No. 17-7410057).

\section{References}

1. Efimtseva E.V. et al. (2007) Disaccharide nucleosides and their incorporation into oligonucleotides. Curr. Org. Chem. 11(4):337-354.

2. Mikhailov S.N. et al. (2008) Synthesis of 2'-O- $\alpha-D-$-ribofuranosyladenosine, monomeric unit of poly (ADP-ribose). Tetrahedron. 64(12):2871-2876. 